

\title{
Porous polymer monolithic columns with gold nanoparticles as an intermediate ligand for the separation of proteins in reverse phase-ion exchange mixed mode
}

\author{
Lydia Terborg ${ }^{\text {a }}$, Jorge C. Masini ${ }^{\text {b }}$, Michelle Lin ${ }^{\text {c }}$, Katriina Lipponen ${ }^{\text {d, }}$ \\ Marja-Liisa Riekolla ${ }^{d}$, Frantisek Svec ${ }^{\text {a,* }}$
}

a The Molecular Foundry, E.O. Lawrence Berkeley National Laboratory, Berkeley, CA 94720, United States

${ }^{\mathrm{b}}$ Institute of Chemistry, Department of Fundamental Chemistry, University of São Paolo, C.P. 26077, 05513-970 São Paulo, Brazil

${ }^{\mathrm{c}}$ Department of Chemistry, University of California, Berkeley, CA 94720, United States

${ }^{\mathrm{d}}$ Laboratory of Analytical Chemistry, Department of Chemistry, P.O. Box 55, FIN-00014, University of Helsinki, Helsinki, Finland

\section{A R T I C L E I N F O}

Article history:

Received 28 September 2014

Received in revised form 15 October

2014

Accepted 16 October 2014

Available online 4 November 2014

\section{Keywords:}

Gold nanoparticles

Mixed mode

Monolith

Proteins

Separation

\begin{abstract}
A B S T R A C T
A new approach has been developed for the preparation of mixed-mode stationary phases to separate proteins. The pore surface of monolithic poly(glycidyl methacrylate-co-ethylene dimethacrylate) capillary columns was functionalized with thiols and coated with gold nanoparticles. The final mixed mode surface chemistry was formed by attaching, in a single step, alkanethiols, mercaptoalkanoic acids, and their mixtures on the free surface of attached gold nanoparticles. Use of these mixtures allowed fine tuning of the hydrophobic/hydrophilic balance. The amount of attached gold nanoparticles according to thermal gravimetric analysis was $44.8 \mathrm{wt} . \%$. This value together with results of frontal elution enabled calculation of surface coverage with the alkanethiol and mercaptoalkanoic acid ligands. Interestingly, alkanethiols coverage in a range of 4.46-4.51 molecules $/ \mathrm{nm}^{2}$ significantly exceeded that of mercaptoalkanoic acids with $2.39-2.45$ molecules $/ \mathrm{nm}^{2}$. The mixed mode character of these monolithic stationary phases was for the first time demonstrated in the separations of proteins that could be achieved in the same column using gradient elution conditions typical of reverse phase (using gradient of acetonitrile in water) and ion exchange chromatographic modes (applying gradient of salt in water), respectively.
\end{abstract}

(C) 2014 Production and hosting by Elsevier B.V. on behalf of Cairo University.

\footnotetext{
* Corresponding author. Tel./fax: +1 5104867964.

E-mail address: fsvec@lbl.gov (F. Svec).

Peer review under responsibility of Cairo University.

\section{Production and hosting by Elsevier}

\section{Introduction}

Mixed-mode chromatography refers to chromatographic methods that utilize more than one type of interaction between the stationary phase and analytes in order to achieve their separation [1,2]. While early chromatographic methods preferred stationary phases with a strictly singular functionality, 
packings accommodating a plurality of functionalities turned out to be very useful. They support simultaneous interactions of various types, thus enabling the retention and separation of a multiplicity of compounds in a single run. This advantage of mixed-mode packings over single mode separation media caught attention of the pharmaceutical and biotechnology industries. As a result, mixed-mode chromatography became a popular tool in quality control and downstream processing, and a number of mixed mode columns combining reversed phase, hydrophobic interaction, hydrophilic interaction, and different ion exchange mechanisms are now available from industrial sources.

Mixed-mode chromatography, including both reverse phase and ion exchange mechanisms, has been known for 20 years [3]. The separation of analytes is achieved through the differences in the hydrophobicity and charge of the separated species. The early mixed-mode approaches were achieved by mixing two types of particulate separation media, each with a single chemistry, and packing the mixture into a column. Alternatively, silica with reduced coverage of hydrophobic ligands that still contained free uncapped acidic silanols, i.e. mixed two different ligands in a single packing, was also used. More sophisticated stationary phases were prepared using molecules that contain ion exchange functionality as a part of the hydrophobic ligand. Depending on the position of the ionizable functionality with respect to the pore surface, these phases can be "embedded", i.e. the functionality is close to the surface and the hydrophobic chain extends in a mobile phase environment, or "tipped" with the functionality at the free end of the hydrophobic chain. These stationary phases excel in reproducibility since their chemistry is defined by the attached molecule, not by the preparation process. The chemistry of all current mixed mode columns is given and once the column is available it cannot be changed. Also, optimizing the column chemistry is a tedious process since it always requires several synthetic steps.

Sulfur containing functionalities are known to strongly interact with noble metals and this property is widely used in analytical chemistry [4-6]. For example, several authors have demonstrated this fact with the placement of gold and silver nanoparticles on the pore surface of polymer-based monolithic supports that are then functionalized with thiols [7-16]. In contrast to porous beads with a maximum pore size typically in the range of low tens of nanometers, monolithic columns feature large through pores, with sizes reaching hundreds of nanometers or even single micrometers. Therefore, the monolithic structures are an ideal support for immobilization of nanoparticles without affecting the permeability for the flow. For example, $15 \mathrm{~nm}$ gold nanoparticles covering a pore with a size of $800 \mathrm{~nm}$ decrease the pore cross section by less than $4 \%$. The large pores also enable convectional mass transfer of large molecules to the interacting site that is much faster than diffusion through the stagnant mobile phase in pores enabling mass transport within beads. Therefore, porous polymer monoliths are almost ideal stationary phase for the rapid separation of proteins.

Our recent studies $[7,8,11,12,14]$ demonstrated that pores of polymer monoliths functionalized with thiol groups can be coated with an almost continuous layer of gold nanoparticles. The simultaneous interaction of the gold nanoparticles with multiple thiol groups on the pore surface is the reason for the high stability of their attachment [7]. However, no matter how many of these attaching interactions occur, for sterical reasons they always occupy only a small fraction of the external surface area of the nanoparticle. The majority of the surface is then available for interactions with small molecules containing thiol groups. This feature makes immobilized gold nanoparticles excellent intermediate ligands that can be used coverage with compounds allowing wide variations in the surface chemistry. In addition, the interaction with a single thiol group is weak enough to enable its stripping and replacing with a different thiol containing molecule. Thus, the chemistry of a single column containing the gold nanoparticles can be changed at any time [8]. To date, all applications where the gold nanoparticles have served as the intermediate ligand were demonstrated with the immobilization of a single type of thiol molecules forming a homogeneous surface chemistry.

In this paper, we present a different approach that extends our previous published work [12]. Our new method facilitates the creation of monolithic columns for mixed mode chromatographic separation of proteins since it permits the functionalization with two different chemistries at the same time. This approach entails the simultaneous attachment of hydrophobic and ionizable molecules on the gold nanoparticles immobilized at the pore surface of a monolith. The proportion of both components during functionalization enables fine tuning of polarity of the surface chemistry. Since the thiols are bound non-covalently, the proportions of the surface chemistries can be changed as needed always using the same column. Our new technique can simplify tailoring stationary phases for specific applications.

\section{Experimental}

\section{Chemicals}

Glycidyl methacrylate (GMA) and ethylene dimethacrylate (EDMA) monomers were obtained from Sigma-Aldrich (St. Louis, MO, USA) and purified by passing them through an aluminum oxide column for removal of the inhibitor. Azobisisobutyronitrile (AIBN), cyclohexanol, 1-dodecanol, hydrochloric acid, sodium hydroxide, acetic acid, trifluoroacetic acid, cystamine dihydrochloride, propylamine, tris(2-carboxylethyl)phosphine hydrochloride (TCEP) solution $(0.5 \mathrm{~mol} / \mathrm{L}$, $\mathrm{pH}=7$ adjusted with ammonium hydroxide), 3-(trimethoxysilyl)propyl methacrylate, tris(hydroxymethyl)aminomethane (TRIS), 1-octanethiol (C8), 1-dodecanethiol (C12), 11-mercaptoundecanoic acid (11MUA), 8-mercaptooctanoic acid (8MOA), 2-diethylaminoethane-thiol hydrochloride (DAM), monobasic and dibasic sodium phosphates, and sodium chloride were obtained from Sigma-Aldrich in the highest quality available. The same vendor also provided the proteins: bovine serum albumin (BSA), lysozyme from chicken egg (LYZ), ribonuclease A from bovine pancreas (RNase A), hemoglobin from bovine blood $(\mathrm{Hb})$, myoglobin from horse skeleton muscle $(\mathrm{Mb})$ and cytochrome $\mathrm{C}$ from bovine heart (Cyt-C). The proteins were dissolved in water prior to chromatographic separation at concentrations ranging from 1.0 to $2.0 \mathrm{mg} / \mathrm{mL}$.

The gold nanoparticles (GNP) with a particle size of $15 \mathrm{~nm}$ were purchased from Ted Pella, Inc (Redding, CA, USA). HPLC-grade solvents (acetonitrile (ACN), acetone, methanol, and ethanol) were received from EMD Chemicals (Gibbstown, NJ, USA). Deionized water with 18.2 $\mathrm{M} \Omega \mathrm{cm}$ from a Barnstead 
Easypure II water purification system (Thermo Fisher Scientific, Waltham, MA, USA) was used throughout this work. Polyimide coated fused silica capillaries with an inner diameter of $100 \mu \mathrm{m}$ were purchased from Polymicro Technologies (Phoenix, AZ, USA).

\section{Instrumentation}

A syringe pump (Kd Scientific, New Hope, PA, USA) was used to pump both the polymerization mixture and the derivatization reagents through the capillary and monolith, respectively. The gold nanoparticles were pumped through the monolithic capillary columns using a high pressure 260D syringe pump (ISCO, Lincoln, NE, USA) equipped with a Rheodyne 7725 manual six-port sample injection valve (Rheodyne, Rohnert Park, CA) and a $2 \mathrm{~mL}$ loop to avoid contamination of the pump with GNP. A 1200 series nanoflow HPLC system from Agilent Technologies (Santa Clara, CA, USA) equipped with a degasser, $80 \mathrm{~nL}$ UV detection flow cell, and an external microvalve injector with a $10 \mathrm{~nL}$ sample loop (Valco Instruments Co Inc., Schenkon, Switzerland) was used for the chromatographic evaluation. Reversed phase separations were carried out using a linear mobile phase gradient from 5 to $70 \mathrm{vol} \%$ acetonitrile in $0.1 \mathrm{vol} \%$ aqueous trifluoroacetic acid in $7.5 \mathrm{~min}$ at a flow rate of $2.0 \mu \mathrm{L} / \mathrm{min}$ and the peaks detected at $210 \mathrm{~nm}$. A linear salt gradient of mobile phase composed of (A) $10 \mathrm{vol} \% \mathrm{ACN}$ in phosphate buffer ( $\mathrm{pH} 7$ ) and (B) $0.5 \mathrm{~mol} / \mathrm{L} \mathrm{NaCl}$ in $\mathrm{A}$ was used for the separation in an ion exchange chromatographic mode. The gradient time from $100 \%$ A to $90 \mathrm{vol} \% \mathrm{~B}$ in A was $1 \mathrm{~min}$ at a flow rate $4 \mu \mathrm{L} / \mathrm{min}$.

An external injector with a $20 \mu \mathrm{L}$ sample loop was used for the frontal analysis. The thiol solution with a concentration of $1-3 \mathrm{~mol} / \mathrm{L}$ in ethanol was pumped through the system at a flow rate of $0.5 \mu \mathrm{L} / \mathrm{min}$ with ethanol as the pushing solvent and the breakthrough was detected at $210 \mathrm{~nm}$.

Scanning electron micrographs were obtained using a Zeiss Gemini Ultra Field-Emission Scanning Electron Microscope (Peabody, MA, USA). The polymer monolith samples were sputtered with gold using the SCD 050 sputter coater (BALTEC AG, Balzers, Lichtenstein). The SEM system equipped with an energy dispersive X-ray (EDX) spectrometer from EDAX (Mahwah, NJ, USA) was used for elemental X-ray analysis.

Thermogravimetric analysis (TGA) using a Q5000IR from TA Instruments (New Castle, DE, USA) was carried out in a temperature range of $35-900^{\circ} \mathrm{C}$ at a heating rate of $5^{\circ} \mathrm{C} / \mathrm{min}$ under a nitrogen atmosphere.

\section{Determination of contents of gold and alkanethiols}

TGA was applied for the determination of the gold content. All capillaries were cut to the same length with a weight of about $5 \mathrm{mg}$. The monolithic polymer decomposed at a temperature of about $360^{\circ} \mathrm{C}$, the polyimide coating then decomposed at $585^{\circ} \mathrm{C}$. The incombustible gold content was calculated using the following equation:

$\mathrm{Au}(\mathrm{wt} \%)=\left[\frac{W+G B-W_{E B}}{W_{G I}-W_{E I}}\right] \times 100$

where $W_{G B}$ is the weight of monolithic capillary column with attached GNP after burning the organic polymer and polyimide coating, $W_{E B}$ is the weight of the empty capillary after burning the coating, $W_{M I}$ is the initial weight of the monolithic capillary column with attached GNP, and $W_{E I}$ is the initial weight of the empty capillary.

The amount of adsorbed thiol was determined from results of frontal analysis using Eq. (2):

Alkanethiol content $/ \mathrm{cm}$ GNP column $=\frac{(i-v) * f * c}{L}$

where $i$ is the elution time at the inflection point of the breakthrough curve, $v$ is the void time determined by injection of non-retained compound, $f$ is the flow rate, $c$ is the concentration of the thiol, and $L$ is the column length. A simple calculation then provided the number of thiol molecules per square nanometer of the external surface of nanoparticle.

\section{Preparation of monolithic capillary columns}

The preparation of generic poly(glycidyl methacrylate-coethylene dimethacrylate) monoliths and their modification with cystamine, tris(2-carboxylethyl)phosphine, and $15 \mathrm{~nm}$ gold nanoparticles shown in Fig. 1 was carried out using the procedure developed by Lv et al. [12]. The inner surface of the capillary was first vinylized using 3-(trimethoxysilyl)propyl methacrylate to enable covalent attachment of the monolith. The polymerization mixture that consisted of $30 \mathrm{wt} \% 1$-dodecanol and $30 \mathrm{wt} \%$ cyclohexane (both porogens), $24 \mathrm{wt} \%$ glycidyl methacrylate and $16 \mathrm{wt} \%$ ethylene dimethacrylate (both monomers) and AIBN as initiator ( $1 \mathrm{wt} \%$ with respect to monomers) was homogenized by sonication for $15 \mathrm{~min}$ and degassed by purging with nitrogen for $5 \mathrm{~min}$. The polymerization mixture was then introduced into the vinylized capillary. The capillary was sealed at both ends with a rubber septum and immersed for $24 \mathrm{~h}$ in a water bath thermostated at $60{ }^{\circ} \mathrm{C}$. After the polymerization reaction was completed, a piece of capillary containing the monolith was cut at both ends of the capillary to liberate the virgin monolithic structure, and the monolith was flushed with acetonitrile to remove unreacted components.

Subsequently the generic monoliths were modified with cystamine and TCEP to generate the desired thiol functionalities required for attachment of the gold nanoparticles [12]. A high pressure 260D ISCO syringe pump was used for the modification of the monoliths with GNP. Colloidal dispersion of GNP was filled in the loop and the dispersion contained in the loop was pumped through the functionalized monolithic column at a flow rate of $5 \mu \mathrm{L} / \mathrm{min}$ until the entire column length turned deep red and a pink solution was observed coming out of the capillary outlet.

The last step in the preparation of the functionalized monoliths included pumping $500 \mu \mathrm{L}$ of $\mathrm{C} 8$ and $\mathrm{C} 12$ alkanethiol solution $(3.0 \mathrm{~mol} / \mathrm{L}$ in ethanol) at a flow rate of $0.5 \mu \mathrm{L} / \mathrm{min}$ through the monoliths. This process resulted in the reversed phase columns with respective to $\mathrm{C} 8$ and $\mathrm{C} 12$ functionalities. "Tipped" columns with ion exchange functionalities were obtained via pumping respective solutions of mercaptoalkanoic acids (8MOA, 11MUA, $0.5 \mathrm{~mol} / \mathrm{L}$ in ethanol) and the aminothiol (DAM $0.5 \mathrm{~mol} / \mathrm{L}$ in TRIS buffer $\mathrm{pH}$ 8). Columns containing combined alkyl and alkylcarboxylic acid ligands were prepared by pumping mixed solution of alkanethiols and mercaptoalkanoic acids (molar ratios 1:3, 1:1, and 3:1) 
<smiles>C=C(C)C(=O)OCC1OC1=CC(=O)C(C)=CCOC(=O)C(C)=CCCC</smiles><smiles>CC(=O)OCCNCCSSCCN</smiles><smiles>CCC(=O)OCC(O)CNCCSSCCN</smiles>

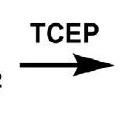<smiles>CC(=O)OCC(O)CNCCS</smiles><smiles>CCCCCC(C)OCC(O)CNCCS</smiles><smiles>[R]CC[C@H](C)C(C)C</smiles><smiles>[R]CCSC1CCCCCC1CSCCNCCC(O)COC(C)=O</smiles>

Fig. 1 Preparation of poly(glycidyl methacrylate-co-ethylene dimethacrylate) monolith and its modifications with cystamine, reduction with tris(2-carboxylethyl)phosphine, attachment of gold nanoparticles, and coating with 1-octanethiol.

through the GNP functionalized monoliths. All the columns were then washed with ethanol and acetonitrile.

\section{Results and discussion}

\section{Preparation of monolithic columns with GNP}

Epoxide functionalities of the generic poly(glycidyl methacrylate-co-ethylene dimethacrylate) monolith reacted first with cystamine, and the monolith was subsequently treated with TCEP to cleave the disulfide bridges of cystamine and liberate the desired free thiol groups. GNP dispersion was then pumped through the monolithic column and the $15 \mathrm{~nm}$ nanoparticles were attached to the pore surface. The monolith changed its color from white to pinkish-red during this process. The SEM image in Fig. 2 confirms the excellent pore surface coverage with GNP. Elemental analysis using EDX indicated the presence of $44.8 \mathrm{wt} \%$ gold. TGA analysis confirmed this value. This gold content is on par with $45.0 \mathrm{wt} \%$ that we found previously [12].

The open surface of GNP was then functionalized using alkanethiols, mercaptoalkanoic acids, and amine containing

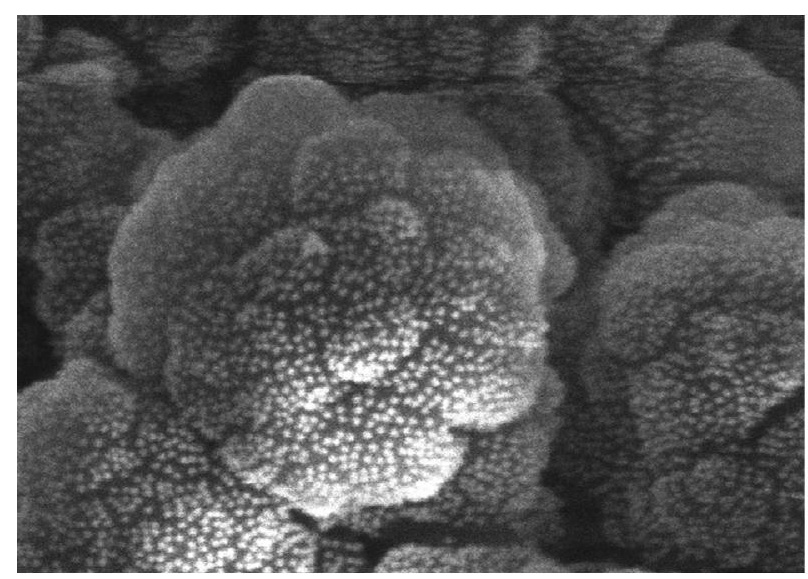

Fig. 2 Scanning electron micrograph of the internal structures of functionalized poly(glycidyl methacrylate-co-ethylene dimethacrylate) monoliths with attached $15 \mathrm{~nm}$ gold nanoparticles. thiols to obtain monolithic capillary columns suitable for separations using reverse phase, cation exchange, and anion exchange mechanisms. First, frontal elution was used to characterize the saturation rate and quantity of the thiol molecules attached to the GNP surface. Fig. 3 shows an example of the breakthrough curve for the column through which an ethanol solution of 11MUA was pushed at a flow rate of $2 \mu \mathrm{L} / \mathrm{min}$ using the precise nano HPLC pump. The breakthrough curve is very steep and confirms that both the mass transport and the interaction rates of the thiols with the GNP surface were very fast.

Scanning electron micrographs confirmed that the GNP has a spherical shape. A simple calculation for GNP with a diameter of $15 \mathrm{~nm}$ results in a surface area of $706.86 \mathrm{~nm}^{2}$ and a volume of $1767.15 \mathrm{~nm}^{3}$. Both EDX and TGA determined $44.8 \mathrm{wt} \%$ gold in the monolith. Using density of gold $19.3 \mathrm{~g} /$ $\mathrm{cm}^{3}$, we calculated that $1 \mathrm{~cm}$ of the monolith contains about $7.7 \times 10^{11}$ gold nanoparticles. Knowing the number of GNP attached in the column, the concentration of the thiol molecules in the solution, and the volume at the $10 \%$ breakthrough,

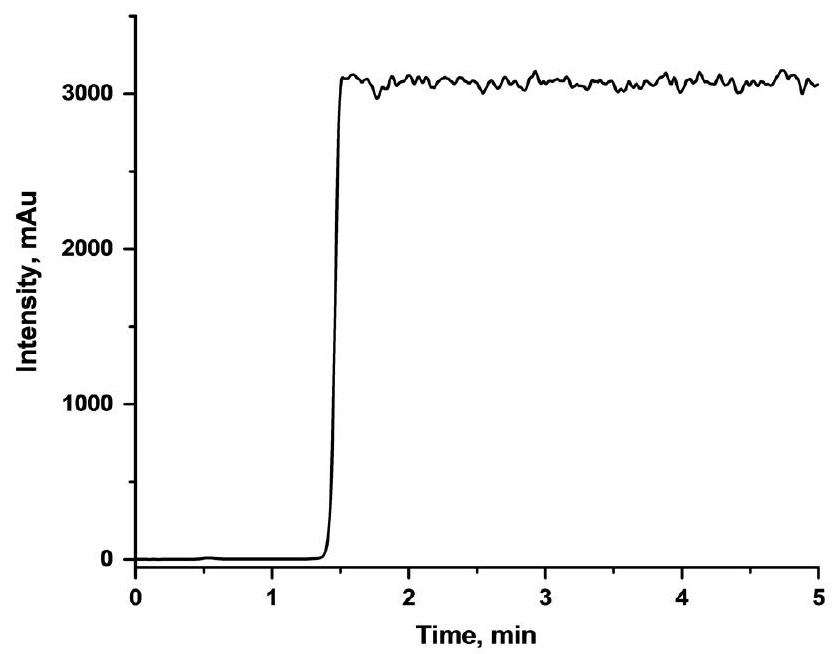

Fig. 3 Breakthrough curve of 11-mercaptoundecanoic acid in gold nanoparticles containing monolithic column. Conditions: column $12 \mathrm{~cm} \times 100 \mu \mathrm{m}$; Flow rate: $0.5 \mu \mathrm{L} / \mathrm{min}$; Concentration of ethanolic solution of the acid $21.8 \mu \mathrm{g} / \mu \mathrm{L}$; UV detection at $210 \mathrm{~nm}$. 
Table 1 Calculated numbers of the thiol-containing molecules per unit of surface area of gold nanoparticles attached to the pore surface within monolith.

\begin{tabular}{ll}
\hline Thiol-containing compound & Molecules per $\mathrm{nm}^{2}$ \\
\hline 1-Octanethiol & 4.51 \\
1-Dodecanethiol & 4.46 \\
8-Mercaptooctanoic acid & 2.45 \\
11-Mercaptoundecanoic acid & 2.39 \\
2-Diethylaminoethanethiol.HCl & 4.69 \\
\hline
\end{tabular}

we were able to calculate the number of thiol molecules per unit of the external surface area of GNP.

Table 1 presents these values for all individual compounds used in this study. The average numbers 4.51 and 4.46 molecules per $\mathrm{nm}^{2}$ of GNP surface were close for both C8 and $\mathrm{C} 12$ linear alkanethiols. These numbers were somewhat lower than those found in the literature for similar compounds [17] since the alkanethiols cannot bind to the gold surface that is already used for the attachment to the pore surface of the solid support. They also showed that an increase in the length of the alkyl had only a negligible effect on the number of attached molecules. The same observation, i.e. small effect of the alkyl length, also applied for C7 and C10 mercaptoalkanoic acids. In contrast to alkanethiols, the numbers of 8-mercaptooctanoic and 11-mercaptoundecanoic acid molecules attached to the GNP were 2.45 and 2.39 per $\mathrm{nm}^{2}$ of
GNP surface, respectively, and represent only slightly more than one half of the coverage observed with alkanethiols. We speculate that the repulsive effects of the ionized carboxylic acid functionalities prevent denser coverage of the gold surface with these molecules. Interestingly, this inference appears not to apply to coverage with diethylaminoethanethiol that was 4.69 molecules per $\mathrm{nm}^{2}$. This value was the highest of all the molecules we tested. This may be result of both the non-ionic character of the hydrochloride form and the smaller molecular weight of the amine molecule.

In order to modify several columns at the same time, and to increase the production throughput, in follow-up experiments we used a syringe pump operating several syringes simultaneously to push the thiol solutions through the monoliths. The flow rate was attenuated to $0.5 \mu \mathrm{L} / \mathrm{min}$ to prevent pressure buildup in the system. We did not find any difference in performance of columns functionalized using either approach.

\section{Separation of proteins}

The aim of this study was to develop a technique enabling design of separation media in which the chemistry can be easily adjusted for each specific target protein mixture. Several control experiments were carried out to demonstrate the effect of the functionalized gold nanoparticles on the separation performance. As presented in Table 2, the generic column retained and then separated all three proteins RNase A, Cyt-C, and $\mathrm{Mb}$ present in the test mixture. This ability to separate can be

Table 2 Reverse phase retention factors of selected proteins using monolithic capillary columns varying in modifications in. Conditions: column: $12 \mathrm{~cm} \times 100 \mu \mathrm{m}$ i.d.; linear mobile phase gradient $5-70 \%$ acetonitrile in 0.1 vol\% aqueous trifluoroacetic acid in $7.5 \mathrm{~min}$; Flow rate $2.0 \mu \mathrm{L} / \mathrm{min}$; UV detection $210 \mathrm{~nm}$.

\begin{tabular}{|c|c|c|c|c|c|c|}
\hline \multirow[t]{3}{*}{ Functionalization with } & \multicolumn{6}{|c|}{ Retention factor, $k$} \\
\hline & \multicolumn{3}{|c|}{ Mixture 1} & \multicolumn{3}{|c|}{ Mixture 2} \\
\hline & RNase $A^{a}$ & Cyt-C & $\mathrm{Mb}$ & LYZ & BSA & $\mathrm{Hb}$ \\
\hline \multicolumn{7}{|l|}{ Control experiments } \\
\hline None (generic) & 6.6 & 7.7 & 8.6 & $\mathrm{~N} / \mathrm{A}^{\mathrm{b}}$ & $\mathrm{N} / \mathrm{A}$ & $\mathrm{N} / \mathrm{A}$ \\
\hline Cystamine & 0.9 & 0.9 & 0.9 & $\mathrm{~N} / \mathrm{A}$ & $\mathrm{N} / \mathrm{A}$ & $\mathrm{N} / \mathrm{A}$ \\
\hline TCEP & 1.0 & 1.0 & 1.0 & $\mathrm{~N} / \mathrm{A}$ & $\mathrm{N} / \mathrm{A}$ & $\mathrm{N} / \mathrm{A}$ \\
\hline Gold nanoparticles & 1.0 & 1.0 & 1.0 & $\mathrm{~N} / \mathrm{A}$ & $\mathrm{N} / \mathrm{A}$ & $\mathrm{N} / \mathrm{A}$ \\
\hline \multicolumn{7}{|c|}{ Columns with attached and functionalized gold nanoparticles } \\
\hline $\mathrm{C}-8$ & 6.9 & 7.3 & 8.8 & 7.8 & 8.3 & 8.6 \\
\hline 11MUA:C8 1:3 & 5.7 & 6.2 & 7.1 & 6.7 & 7.0 & 7.2 \\
\hline 11MUA:C8 1:1 & 5.6 & 6.1 & 7.1 & 6.6 & 7.3 & 7.5 \\
\hline 11MUA:C8 3:1 & 5.3 & 6.0 & 6.9 & 0 & 6.8 & 6.9 \\
\hline 11-MUA & 4.5 & 5.3 & 6.1 & 0 & 6.0 & 6.4 \\
\hline $\mathrm{C}-12$ & 6.8 & 7.4 & 8.4 & 7.7 & 8.2 & 8.6 \\
\hline 11MUA:C12 1:3 & 6.5 & 7.1 & 8.4 & 6.9 & 7.5 & 7.7 \\
\hline 11MUA:C12 1:1 & 6.6 & 7.2 & 8.3 & 7.7 & 8.2 & 8.4 \\
\hline 11MUA:C12 3:1 & 6.1 & 6.6 & 7.7 & 7.0 & 7.7 & 7.7 \\
\hline 11-MUA & 4.5 & 5.3 & 6.1 & 0 & 6.0 & 6.4 \\
\hline C-8 & 6.9 & 7.3 & 8.8 & 7.8 & 8.3 & 8.6 \\
\hline 8MOA:C8 1:3 & 6.2 & 6.7 & 7.8 & 7.2 & 7.8 & 8.3 \\
\hline 8MOA:C8 1:1 & 6.3 & 6.8 & 7.7 & 7.0 & 7.8 & 8.1 \\
\hline 8MOA:C8 3:1 & 5.8 & 6.2 & 7.3 & 6.8 & 7.3 & 7.5 \\
\hline 8-MOA & 0 & 0 & 5.0 & 0 & 1.1 & 5.0 \\
\hline DAM & 4.8 & 5.3 & 6.3 & $\mathrm{~N} / \mathrm{A}$ & $\mathrm{N} / \mathrm{A}$ & $\mathrm{N} / \mathrm{A}$ \\
\hline
\end{tabular}




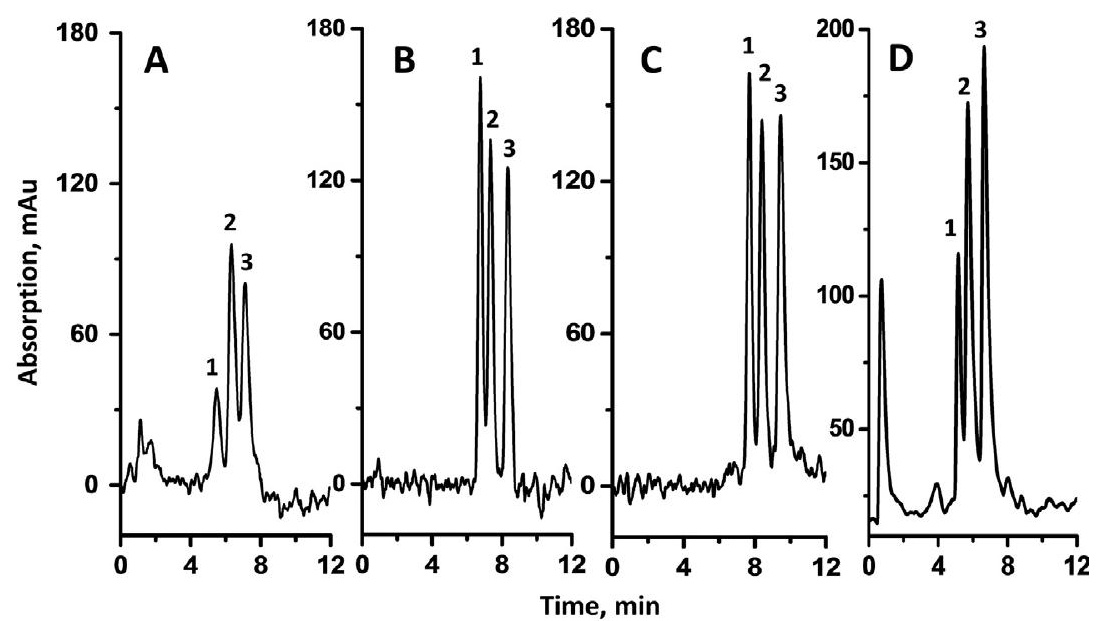

Fig. 4 Reverse phase separation of a mixture of three proteins using differently functionalized gold nanoparticles containing monolithic columns. (A) Column functionalized with 11-mercaptoundecanoic acid; (B): column functionalized with a 1:1 mixture of 11mercaptoundecanoic acid and 1-octanethiol; (C) column functionalized with 1-octanethiol; (D) column functionalized with 2diethylaminoethanethiol; column: $12 \mathrm{~cm} \times 100 \mu \mathrm{m}$; mobile phase: gradient of $5-70 \%$ acetonitrile in $0.1 \%$ aqueous trifluoroacetic acid in $7.5 \mathrm{~min}$; Flow rate: $2 \mu \mathrm{L} / \mathrm{min}$ : UV detection at $210 \mathrm{~nm}$. Peaks: 1 - ribonuclease A, 2 - cytochrome C, 3 - myoglobin.

ascribed to the intrinsic hydrophobicity of the poly(glycidyl methacrylate-co-ethylene dimethacrylate) monolith. During modification with cystamine, TCEP, and GNP, the hydrophobic character of the monolith completely disappeared and the proteins were eluted at the void volume.

Surface chemistry of the columns containing gold nanoparticles was modulated by reaction with individual thiols and their mixtures. The retention factors calculated for differently functionalized columns from separations of six proteins under the reverse phase conditions are summarized in Table 2. These six proteins were divided into two groups of three (RNase A, Cyt-C, Mb, and LYZ, BSA, Hb), and each group was injected separately. The results confirmed that columns modified with C8 and C12 alkanethiols exhibited the highest hydrophobicity and longest retention for all of the proteins. Admixing carboxylic acid derivatives in the surface coverage led to a decrease in retention with an increasing proportion of this compound in the modification mixture. Due to reduced coverage with the mercaptoalkanoic acids described earlier in this text, the effect of these molecules appeared only after they prevailed in the modification mixture, i.e. at the ratios 3:1. The retention reached the lowest level for the singular "tipped" type mixed mode columns obtained after modifications with individual mercaptoundecanoic and mercaptooctanoic acids, respectively. The presence of hydrophilic carboxylic acid groups decreased hydrophobicity of the ligands. However, the elution order of the proteins did not change in any of these columns.

Fig. 4 demonstrates the effect of the presence of carboxylic acid groups, or mixed mode mechanism, with separation of a mixture of RNase A, Cyt-C and $\mathrm{Mb}$. Chromatogram in Fig. 4A represents separation using the mobile phase typical of reverse phase achieved with a column modified with 11-mercaptoundecanoic acid alone. The separation was poor, the peaks were broad, and the retention times short. Both the enhanced surface hydrophilicity and limited surface coverage with the ligand may be responsible for that. The situation improved for columns modified with a 1:1 mixture of 11-mercaptoundecanoic acid and 1-octanethiol (Fig. 4B). The best separation shown in Fig. $4 \mathrm{C}$ and the longest retention times were achieved with the columns modified with pure 1-octanethiol. The last panel D of Fig. 4 presents separations using columns functionalized with 2-diethylaminoethanethiol. This separation was significantly better than that shown in Fig. 4A although both columns were modified with ionizable molecules. The better column performance after functionalization with the amine was probably the result of the much higher surface coverage as discussed above.

We also tested the separation performance of the columns under conditions typical for ion exchange mode, i.e. using an increasing gradient of salt concentration in aqueous mobile phase. Since the stationary phases were operating in the mixed mode, the hydrophobic interactions could not be neglected and

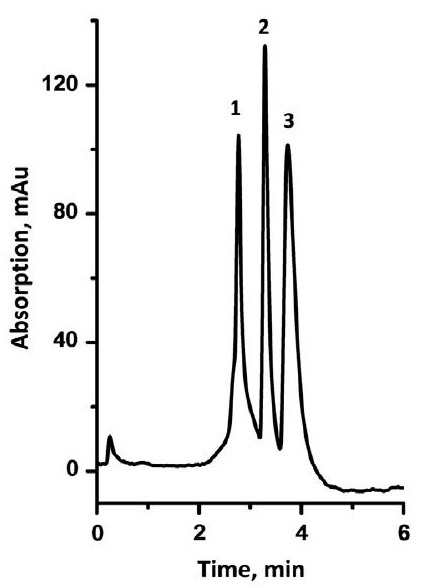

Fig. 5 Weak anion exchange separation of a mixture of three proteins using gold nanoparticles containing monolithic columns functionalized with 8-mercaptooctanoic acid. Conditions: column: $12 \mathrm{~cm} \times 100 \mu \mathrm{m}$; mobile phase A: $10 \mathrm{vol} \%$ acetonitrile in $1 \mathrm{mmol} /$ L sodium phosphate buffer ( $\mathrm{pH}$ 7); $\mathrm{B}: 0.5 \mathrm{~mol} \mathrm{NaCl}$ solution in A; Gradient: $0 \%$ B to $90 \%$ B in $1 \mathrm{~min}$; Flow rate: $4 \mu \mathrm{L} / \mathrm{min}$; UV detection at $210 \mathrm{~nm}$. Peaks: 1 - ribonuclease A, 2 - cytochrome C, 3 - lysozyme. 
Table 3 Effect of surface functionalities on the retention factor of three proteins under conditions typical of ion exchange chromatography. Conditions: column: $12 \mathrm{~cm} \times 100 \mu \mathrm{m}$; mobile phase A: $10 \mathrm{vol} \%$ acetonitrile in $1 \mathrm{mmol} / \mathrm{L} \quad \mathrm{NaH}_{2} \mathrm{PO}_{4} / \mathrm{Na}_{2} \mathrm{HPO}_{4}$ buffer (pH 7); B: $0.5 \mathrm{~mol} / \mathrm{L}$ $\mathrm{NaCl}$ solution in A; gradient: $0 \% \mathrm{~B}$ to $90 \% \mathrm{~B}$ in $1 \mathrm{~min}$; flow rate: $4 \mu \mathrm{L} / \mathrm{min}$; UV detection at $210 \mathrm{~nm}$.

\begin{tabular}{lllll}
\hline Functionality & Molar ratio & RNase $^{\mathrm{a}}$ & Cyt-C & LYZ \\
\hline C8 & & N/E & N/E & N/E \\
C12 & & N/E & N/E & N/E \\
C8:8MOA & $1: 1$ & N/E & N/E & N/E \\
C8:8MOA & $1: 3$ & 8.3 & N/E & 11.8 \\
C8:11MUA & $1: 1$ & $\mathrm{~N} / E$ & N/E & N/E \\
C8:11MUA & $1: 3$ & 8.7 & N/E & 14.7 \\
C12:11MUA & $1: 3$ & N/E & N/E & N/E \\
11MUA & & 8.7 & N/E & 13.3 \\
8MOA & & 8.7 & 13.0 & 14.7 \\
\hline
\end{tabular}

${ }^{a}$ For explanation of protein abbreviations see Experimental.

${ }^{b} \mathrm{~N} / \mathrm{E}=$ no elution was observed under these conditions.

the mobile phase also had to contain strong organic solvent. All three proteins RNase A (pI 9.6), Cyt-C (pI 10.0-10.5), and LYZ (pI 9.3) held a positive net charge in buffer solutions with a $\mathrm{pH}$ value less than 9. Therefore, we optimized the mobile phase. Best results were achieved with a mobile phase comprised of a sodium phosphate buffer ( $\mathrm{pH} \mathrm{7)}$ and $10 \mathrm{vol} \%$ acetonitrile, and the elution was obtained in a gradient of sodium chloride. For example, Fig. 5 shows a good separation of three proteins that was carried out at a high flow rate of $4 \mu \mathrm{L} / \mathrm{min}$, and completed in less than $4 \mathrm{~min}$. It is worth noting that this high speed separation was possible due to the advantageous porous structure of the monolith. Table 3 summarizes the results obtained using ion exchange separation conditions for several columns differing in surface chemistry. Due to the obvious hydrophobic character of most of these mixed mode phases, no elution was observed for any of the proteins until the gold surface was highly saturated with the mercaptoalkanoic acid. However, even then, the retention was substantial with retention factors significantly exceeding those monitored for separations under reverse phase conditions which are presented in Table 2. This observation once again confirms the mixed mode character of the monolithic columns.

\section{Conclusions}

This preliminary report shows that monolithic capillary columns can be designed and prepared in a way that they contain well-defined combination of different chemistries suitable for chromatographic separations of proteins in the mixed mode. This study also demonstrated that monolithic capillary columns, containing gold nanoparticles as an intermediate ligand, enabled simultaneous attachment of two different types of thiol group containing molecules, including plain hydrophobic, and alkylcarboxylic acid moieties. Our results confirmed that the selectivity of these columns can be modulated by adjusting the composition of the alkanethiol/mercaptoalkanoic acid mixture. Good separations of protein mixtures could be then achieved using conditions typical of the gradient elution in the reverse phase and ion exchange modes, respectively.
While these results are promising and open new avenues to the formation of stationary phases including multiplicity of chemistries designed for specific separation, much remains to be done. We are currently focusing our attention on the preparation of mixed mode phases that will be also useful for the separation of small molecules.

\section{Conflict of interest}

The authors have declared no conflict of interest.

\section{Compliance with Ethics Requirements}

This article does not contain any studies with human or animal subjects.

\section{Acknowledgment}

All experimental and characterization work was performed at the Molecular Foundry, Lawrence Berkeley National Laboratory supported by the Office of Science, Office of Basic Energy Sciences, Scientific User Facilities Division of the U.S. Department of Energy, under Contract No. DE-AC02-05CH11231. JCM acknowledges a fellowship from São Paulo Research Foundation (process 2012/14472-9) for funding his three months stay at LBNL.

\section{References}

[1] Yang Y, Geng X. Mixed-mode chromatography and its applications to biopolymers. J Chromatogr A 2011;1218:8813-25.

[2] Zhao G, Dong XY, Sun Y. Ligands for mixed-mode protein chromatography: principles, characteristics and design. J Biotechnol 2009;144:3-11.

[3] McLaughlin LW. Chem Rev 1989;89:309-19.

[4] Sykora D, Kasicka V, Miksik I, Rezanka P, Zaruba K, Matejka P, Kral V. Application of gold nanoparticles in separation sciences. J Sep Sci 2010;33:372-87.

[5] Zhong Z, Male KB, Luong JHT. More recent progress in the preparation of $\mathrm{Au}$ nanostructures, properties, and applications. Anal Lett 2003;36:3097-118.

[6] Guihen E, Glennon JD. Nanoparticles in separation science. Recent developments. Anal Lett 2014;36:3309-36.

[7] Xu Y, Cao Q, Svec F, Fréchet JMJ. Porous polymer monolithic column modified with gold nanoparticles for fishing-out and separation of cysteine-containing peptides. Anal Chem 2010;82:3352-8.

[8] Cao Q, Xu Y, Liu F, Svec F, Fréchet JMJ. Polymer monoliths with exchangeable chemistries: use of gold nanoparticles as intermediate ligands for capillary columns with varying surface functionalities. Anal Chem 2010;82:7416-21.

[9] Connolly D, Twamley B, Paull B. High-capacity gold nanoparticle functionalised polymer monoliths. Chem Commun 2010;46:2109-11.

[10] Alwael H, Connolly D, Clarke P, Thompson R, Twamley B, O'Connor B, Paull B. Pipette-tip selective extraction of glycoproteins with lectin modified gold nanoparticles on a polymer monolithic phase. Analyst 2011;136:2619-28.

[11] Guerrouache M, Mahouche-Chergui S, Chehimi MM, Carbonnier B. Site-specific immobilisation of gold nanoparticles on a porous monolith surface by using a 
thiolyne click photopatterning approach. Chem Commun 2012;48:7486-8.

[12] Lv Y, Maya A, Fréchet JMJ, Svec F. Preparation of porous polymer monoliths featuring enhanced surface coverage with gold nanoparticles. J Chromatogr A 2012;1261:121-8.

[13] Currivan S, Connolly D, Paull B. Production of polymer monolithic capillary columns with integrated gold nanoparticle modified segments for on-capillary extraction. Microchem J 2013;111:32-9.

[14] Lv Y, Lin Z, Tan T, Svec F. Preparation of reusable bioreactors using reversible immobilization of enzyme on monolithic porous polymer support with attached gold nanoparticles. Biotech Bioeng 2014;111:50-8.
[15] Sedlacek O, Kucka J, Svec F, Hruby M. Silver-coated monolithic columns for separation in radiopharmaceutical applications. J Sep Sci 2014;37:798-802.

[16] Floris P, Twamley B, Nesterenko PN, Paull B, Connolly D. Fabrication and characterisation of gold nano-particle modified polymer monoliths for flow-through catalytic reactions and their application in the reduction of hexacyanoferrate. Microchem Acta 2014;181:249-56.

[17] Hinterwirth H, Kappel S, Waitz T, Prohaska T, Lindner W, Lämmerhofer M. Quantifying thiol ligand density of selfassembled monolayers on gold nanoparticles by inductively coupled plasma-mass spectrometry. ACS Nano 2013;7:1129-36. 